University of Nebraska - Lincoln

DigitalCommons@University of Nebraska - Lincoln

Faculty Publications, Department of Statistics

Statistics, Department of

10-2006

\title{
The Exact Distribution of the Multilook Magnitude
}

Saralees Nadarajah

University of Nebraska-Lincoln, saralees.nadarajah@manchester.ac.uk

Samuel Kotz

George Washington University

Follow this and additional works at: https://digitalcommons.unl.edu/statisticsfacpub

Part of the Statistics and Probability Commons

Nadarajah, Saralees and Kotz, Samuel, "The Exact Distribution of the Multilook Magnitude" (2006). Faculty Publications, Department of Statistics. 4.

https://digitalcommons.unl.edu/statisticsfacpub/4

This Article is brought to you for free and open access by the Statistics, Department of at DigitalCommons@University of Nebraska - Lincoln. It has been accepted for inclusion in Faculty Publications, Department of Statistics by an authorized administrator of DigitalCommons@University of Nebraska - Lincoln. 


\title{
The Exact Distribution of the Multilook Magnitude
}

\author{
Saralees Nadarajah and Samuel Kotz
}

\begin{abstract}
Gierull provides a statistical analysis of multilook synthetic aperture radar interferograms. Various expressions for the probability density function, cumulative distribution function, and the moments of associated statistics are derived. It appears, however, that most of these expressions are based on some approximation. In this letter, the corresponding expressions are derived in their exact form, including some elementary representations for certain expressions given by Gierull. A numerical comparison of the exact and approximate expressions is provided.
\end{abstract}

Index Terms-Appell function, Gauss hypergeometric function, interferogram's phase, multilook magnitude, synthetic aperture radar $(\mathbf{S A R})$ interferograms.

\section{INTRODUCTION}

G IERULL [1] examines the statistics of the phase and magnitude of multilook synthetic aperture radar (SAR) interferograms toward deployment of along-track interferometry (ATI) for slow ground moving-target indication (GMTI) and derives various expressions for the probability density function (pdf), cumulative distribution function (cdf), and the moments of the statistics. Most of these expressions are based on certain approximations, whereas others involve nonstandard functions such as the Gauss hypergeometric function, which is defined as

$$
{ }_{2} F_{1}(a, b ; c ; x)=\sum_{k=0}^{\infty} \frac{(a)_{k}(b)_{k}}{(c)_{k}} \frac{x^{k}}{k !}
$$

where $(e)_{k}=e(e+1) \cdots(e+k-1)$ denotes the ascending factorial. The parameters $a, b, c$, and $x$ control the nature of the infinite series in (1). The series terminates if $a$ or $b$ is equal to a negative integer or to zero. For $c=-n(n=0,1, \ldots)$, the series is indeterminate if neither $a$ nor $b$ is equal to $-m$ (where $m<n$ and $m$ is a natural number). The series in (1) converges in the unit circle $|x|<1$ and has a branch point at $x=1$. The following conditions apply for convergence on the unit circle. If $0 \leq a+b-c<1$, then the series converges throughout the entire unit circle except at the point $x=1$. If $a+b-c<0$, then the series converges absolutely throughout the entire circle. If $a+b-c \geq 1$, then the series diverges on the entire unit circle. Numerical routines for the computation of (1) are widely available in packages such as Matlab, Maple, and Mathematica.

In this letter, we show that one can actually derive exact expressions and that some of the expressions given in terms of nonstandard functions can be reduced to elementary forms. The outline is given as follows. In Section II, we derive an elementary expression for the marginal pdf of interferogram's phase (compare with [1, eq. (4)], which involves the Gauss

Manuscript received March 27, 2006; revised April 25, 2006.

S. Nadarajah is with the Department of Statistics, University of Nebraska, Lincoln, NE 68583 USA (e-mail: Saralees.Nadarajah@manchester.ac.uk).

S. Kotz is with George Washington University, Washington, DC 20052 USA.

Digital Object Identifier 10.1109/LGRS.2006.877274 hypergeometric function). In Section III, an exact expression for the pdf of the multilook magnitude is derived (compare with [1, eq. (11)], which provides an approximate form). In Section IV, an exact expression for the cdf of the multilook magnitude is derived (compare with [1, eq. (21)], which provides an approximate form involving the Gauss hypergeometric function). In Section V, we derive exact formulas for the moments of the multilook magnitude (compare with [1, eqs. (12) and (19)], which provide some approximate forms). Finally, in Section VI, we illustrate a numerical comparison of the exact and approximate formulas for the moments of the multilook magnitude.

\section{MARginAl PDF OF INTERFERogram's Phase}

The joint pdf of interferogram's magnitude $(\mathcal{E})$ and phase $(\Psi)$ is given as [1, eq. (3)]

$$
f_{\varepsilon, \Psi}(\eta, \psi)=\frac{2 n^{n+1} \eta^{n}}{\pi \Gamma(n)\left(1-\rho^{2}\right)} \exp (-p \eta) K_{n-1}(c \eta)
$$

where $p=-2 n \rho \cos \psi /\left(1-\rho^{2}\right), c=2 n /\left(1-\rho^{2}\right), \rho$ is the complex correlation coefficient, $n$ is the number of looks, $\Gamma(\cdot)$ denotes the gamma function, and $K_{n-1}(\cdot)$ denotes the modified Bessel function of the third kind of order $n-1$. Usually, one defines

$$
K_{\nu}(x)=\frac{\pi\left\{I_{-\nu}(x)-I_{\nu}(x)\right\}}{2 \sin (\nu \pi)}
$$

(when $\nu$ is an integer, the right-hand side should be interpreted as a limit), where $I_{\nu}(\cdot)$ denotes the modified Bessel function of the first kind defined as

$$
I_{\nu}(x)=\frac{x^{\nu}}{2^{\nu} \Gamma(\nu+1)} \sum_{k=0}^{\infty} \frac{1}{(\nu+1)_{k} k !}\left(\frac{x^{2}}{4}\right)^{k} .
$$

Integrating (2) with respect to the magnitude $\eta$, one obtains

$$
\begin{aligned}
f_{\Psi}(\psi)= & \frac{2 n^{n+1}}{\pi \Gamma(n)\left(1-\rho^{2}\right)} \int_{0}^{\infty} \eta^{n} \exp (-p \eta) K_{n-1}(c \eta) d \eta \\
= & \frac{n^{n+1} c^{1-n} \Gamma(2 n)}{\sqrt{\pi} \Gamma(n) \rho^{2}\left(1-\rho^{2}\right) 2^{n} \Gamma(n+3 / 2)} \\
& \times{ }_{2} F_{1}\left(1, \frac{3}{2} ; n+\frac{3}{2} ; 1-\frac{c^{2}}{p^{2}}\right)
\end{aligned}
$$

for $\left|1-c^{2} / p^{2}\right|<1$, which follows by using [2, eq. (2.16.6.3)]. Using [3, eqs. (7.3.1.1), (7.3.1.9), (7.3.1.127), (7.3.2.83)], 
the hypergeometric term in (4) can be simplified to the elementary form

$$
\begin{aligned}
{ }_{2} F_{1} & \left(1, \frac{3}{2} ; n+\frac{3}{2} ; x\right) \\
= & \frac{(-n-1 / 2)_{n}(x-1)^{n-1}}{(1 / 2-n)_{n} x^{n}} \\
\times & {\left[\begin{array}{l}
-1+n x^{-1 / 2} \operatorname{arctanh}(\sqrt{x})+\sum_{k=2}^{n}(-1)^{k-1}\left(\begin{array}{l}
n \\
k
\end{array}\right) \\
\left.\quad \times\left\{\frac{(2 k-3) ! ! x^{-1 / 2}}{2(2 k-2) ! !} \ln \frac{1+\sqrt{x}}{1-\sqrt{x}}+\sum_{l=1}^{k-1} a_{l} x^{l-1}(x-1)^{-l}\right\}\right]
\end{array}\right.}
\end{aligned}
$$

where $x=1-c^{2} / p^{2}(|x|<1)$ and

$$
a_{l}=\frac{(2 k-3) ! !(l-1)}{2^{k-l}(k-1) !} \sum_{j=l}^{k-1}(-1)^{l-j}\left(\begin{array}{c}
k-1 \\
j
\end{array}\right) \frac{(2 j-2 l-1) ! !}{(2 j-1) ! !} .
$$

Here, $(2 m) ! !=2^{m} m !$ and $(2 m+1) ! !=2^{m+1} \pi^{-1 / 2} \Gamma(m+$ $3 / 2)$ with the convention that $(0) ! !=1$. Combining (4) and (5) yields an elementary expression for the marginal pdf of interferogram's phase (compare with [1, eq. (4)]).

\section{PDF of Multilook Magnitude}

Multilook magnitude is defined by the random variable $Z=w \mathcal{E}$, where $w$ and $\mathcal{E}$ are independent random variables specified by the pdfs

$$
f_{W}(w)=\frac{\nu^{\mu}}{\Gamma(\mu)} w^{-\mu-1} \exp \left(-\frac{\nu}{w}\right)
$$

(for $w>0$ ) and

$$
f_{\varepsilon}(\eta)=\frac{4 n^{n+1} \eta^{n}}{\Gamma(n)\left(1-\rho^{2}\right)} I_{0}(b \eta) K_{n-1}(c \eta)
$$

(for $\eta>0$ ), respectively, where $b=2 n \rho /\left(1-\rho^{2}\right), \mu>0$ denotes the degrees of freedom and $\nu>0$ is a shape parameter. Gierull [1] provides an approximate formula for the pdf of $Z$ [1, eq. (11)]. Here, we derive an expression for the pdf of $Z$. First, we write

$$
\begin{aligned}
f_{Z}(z)= & \int_{0}^{\infty} \frac{1}{w} f_{W}(w) f_{\mathcal{E}}\left(\frac{z}{w}\right) d w \\
= & \frac{4 n^{n+1} z^{n} \nu^{\mu}}{\Gamma(\mu) \Gamma(n)\left(1-\rho^{2}\right)} \\
& \times \int_{0}^{\infty} w^{-n-\mu-2} \exp \left(-\frac{\nu}{w}\right) I_{0}\left(\frac{b z}{w}\right) K_{n-1}\left(\frac{c z}{w}\right) d w \\
= & \frac{4 n^{n+1} z^{n} \nu^{\mu}}{\Gamma(\mu) \Gamma(n)\left(1-\rho^{2}\right)} \\
& \times \int_{0}^{\infty} x^{n+\mu} \exp (-\nu x) I_{0}(b z x) K_{n-1}(c z x) d x
\end{aligned}
$$

which follows after substituting $x=1 / w$. Using the definition in (3), one can reexpress (8) as

$$
\begin{aligned}
& f_{Z}(z) \\
& =\frac{2 \pi n^{n+1} z^{n} \nu^{\mu}\{I(n+\mu+1, \nu, b z, c z)-J(n+\mu+1, \nu, b z, c z)\}}{\Gamma(\mu) \Gamma(n)\left(1-\rho^{2}\right) \sin ((n-1) \pi)}
\end{aligned}
$$

where

$$
\begin{aligned}
I(\alpha, p, b, c) & =\int_{0}^{\infty} x^{\alpha-1} \exp (-p x) I_{0}(b x) I_{1-n}(c x) d x \\
J(\alpha, p, b, c) & =\int_{0}^{\infty} x^{\alpha-1} \exp (-p x) I_{0}(b x) I_{n-1}(c x) d x .
\end{aligned}
$$

Application of [2, eq. (2.15.20.2)] shows that one can calculate

$$
\begin{aligned}
I(\alpha, p, b, c)= & \frac{c^{1-n} \Gamma(\alpha+1-n)}{2^{1-n} p^{\alpha+1-n} \Gamma(2-n)} F_{4} \\
& \times\left(\frac{\alpha+1-n}{2}, \frac{\alpha+2-n}{2} ; 1,2-n ; \frac{b^{2}}{p^{2}}, \frac{c^{2}}{p^{2}}\right) \\
J(\alpha, p, b, c)= & \frac{c^{n-1} \Gamma(\alpha+n-1)}{2^{n-1} p^{\alpha+n-1} \Gamma(n)} F_{4} \\
& \times\left(\frac{\alpha+n-1}{2}, \frac{\alpha+n}{2} ; 1, n ; \frac{b^{2}}{p^{2}}, \frac{c^{2}}{p^{2}}\right)
\end{aligned}
$$

where $F_{4}$ denotes the Appell function of the fourth kind defined as

$$
F_{4}\left(a, b ; c, c^{\prime} ; z, \xi\right)=\sum_{k=0}^{\infty} \sum_{l=0}^{\infty} \frac{(a)_{k+l}(b)_{k+l} z^{k} \xi^{l}}{(c)_{k}\left(c^{\prime}\right)_{l} k ! l !} .
$$

Combining (9), (10), and (11), one obtains an exact expression for the pdf of $Z$ in terms of the Appell function. The Appell functions are well known, and numerical routines for their exact computation are available in packages such as Mathematica.

\section{CDF of Multilook Magnitude}

Here, we derive an exact formula for the cdf of $Z$ (compare with the approximate formula given by [1, eq. (21)]). Noting that $Z=w \mathcal{E}$, where $w$ and $\mathcal{E}$ are independent random variables specified by (6) and (7), respectively, one can write

$$
F_{Z}(z)=\int_{0}^{\infty} F_{W}\left(\frac{z}{\eta}\right) f_{\varepsilon}(\eta) d \eta
$$

Because $W$ is a reciprocal of a chi-square random variable, one has [4, Ch. 18]

$$
F_{W}(w)=\exp \left(-\frac{\nu}{w}\right) \sum_{k=0}^{\mu-1} \frac{1}{k !}\left(\frac{\nu}{w}\right)^{k}
$$


Substituting (13) into (12), one obtains

$$
\begin{aligned}
F_{Z}(z)= & \frac{4 n^{n+1}}{\Gamma(n)\left(1-\rho^{2}\right)} \sum_{k=0}^{\mu-1} \frac{1}{k !}\left(\frac{\nu}{z}\right)^{k} \\
& \quad \times \int_{0}^{\infty} \eta^{n+k} \exp \left(-\frac{\nu \eta}{z}\right) I_{0}(b \eta) K_{n-1}(c \eta) d \eta .
\end{aligned}
$$

The integral in (14) is of the same form as that in (8). Thus, (14) can be simplified to

$$
\begin{aligned}
F_{Z}(z)= & \frac{2 \pi n^{n+1}}{\Gamma(n)\left(1-\rho^{2}\right) \sin ((n-1) \pi)} \sum_{k=0}^{\mu-1} \frac{1}{k !}\left(\frac{\nu}{z}\right)^{k} \\
& \times\left\{I\left(n+k+1, \frac{\nu}{z}, b, c\right)-J\left(n+k+1, \frac{\nu}{z}, b, c\right)\right\}
\end{aligned}
$$

where $I(\cdot)$ and $J(\cdot)$ are given by (10) and (11), respectively. Hence, one obtains an exact expression for the cdf of $Z$ in terms of the Appell function of the fourth kind.

\section{Moments of Multilook Magnitude}

Because $Z=w \mathcal{E}$ and $w$ and $\mathcal{E}$ are independent random variables, the $r$ th moment of $Z$ is simply $E\left(Z^{r}\right)=E\left(w^{r}\right) E\left(\mathcal{E}^{r}\right)$. It is well known that the $r$ th moment of a reciprocal of a chisquare random variable is given as [4, Ch. 18]

$$
E\left(W^{r}\right)=\frac{\nu^{r} \Gamma(\mu-r)}{\Gamma(\mu)}
$$

The $r$ th moment of $\mathcal{E}$ can be calculated as

$$
\begin{aligned}
E\left(\varepsilon^{r}\right)= & \frac{4 n^{n+1}}{\Gamma(n)\left(1-\rho^{2}\right)} \int_{0}^{\infty} \eta^{n+r} I_{0}\left(\frac{2 n \rho \eta}{1-\rho^{2}}\right) K_{n-1}\left(\frac{2 n \eta}{1-\rho^{2}}\right) d \eta \\
= & \frac{\left(1-\rho^{2}\right)^{n+r}}{n^{r} \Gamma(n)} \Gamma\left(n+\frac{r}{2}\right) \Gamma\left(1+\frac{r}{2}\right) \\
& \times{ }_{2} F_{1}\left(n+\frac{r}{2}, 1+\frac{r}{2} ; 1 ; \rho^{2}\right)
\end{aligned}
$$

for $|\rho|<1$, which follows by using [2, eq. (2.16.28.1)]. Combining (15) and (16), one obtains the $r$ th moment of $Z$ as

$$
\begin{aligned}
E\left(Z^{r}\right)=\frac{\nu^{r}\left(1-\rho^{2}\right)^{n+r} \Gamma(\mu-r)}{n^{r} \Gamma(n) \Gamma(\mu)} & \Gamma\left(n+\frac{r}{2}\right) \Gamma\left(1+\frac{r}{2}\right) \\
& \times{ }_{2} F_{1}\left(n+\frac{r}{2}, 1+\frac{r}{2} ; 1 ; \rho^{2}\right)
\end{aligned}
$$

for $|\rho|<1$ and $r \geq 1$ (compare with [1, eq. (12)]). In particular, the first two moments of $Z$ are

$$
E(Z)=\frac{\sqrt{\pi} \nu\left(1-\rho^{2}\right)^{n+1}}{2 n \Gamma(n)(\mu-1)} \Gamma\left(n+\frac{1}{2}\right){ }_{2} F_{1}\left(n+\frac{1}{2}, \frac{3}{2} ; 1 ; \rho^{2}\right)
$$

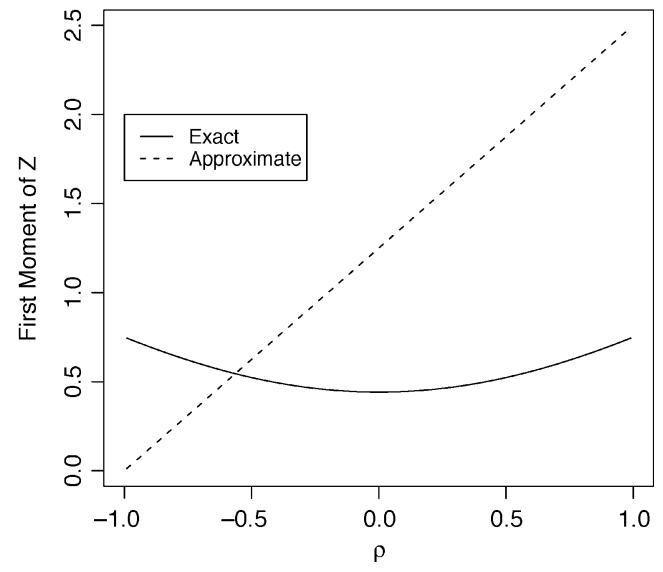

Fig. 1. Comparison of the exact (17) and the approximate (19) expressions for $E(Z)$. It is assumed that $n=2, \mu=5, \nu=3$, and $\rho=-0.99$, $-0.98, \ldots, 0.99$

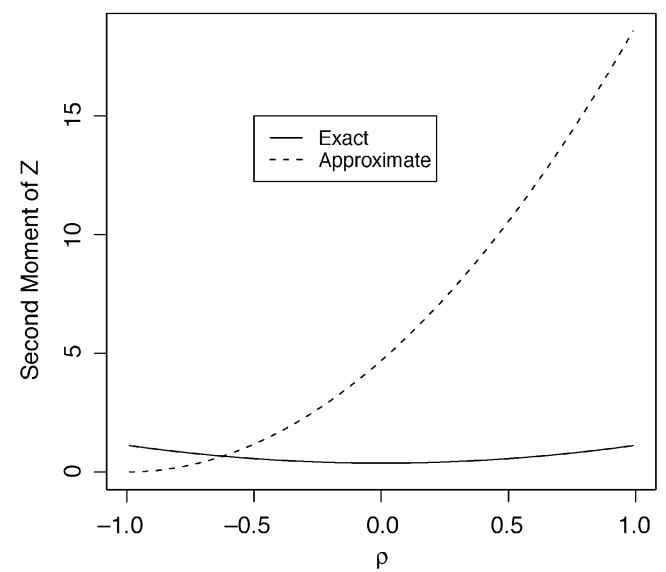

Fig. 2. Comparison of the exact (18) and the approximate (20) expressions for $E\left(Z^{2}\right)$. It is assumed that $n=2, \mu=5, \nu=3$, and $\rho=-0.99$, $-0.98, \ldots, 0.99$.

and

$$
E\left(Z^{2}\right)=\frac{\nu^{2}\left(1+n \rho^{2}\right)}{n(\mu-1)(\mu-2)}
$$

respectively (compare with [1, eq. (19)]), for $|\rho|<1$.

\section{NUMERICAL COMPARISON}

Here, we provide a numerical comparison of the exact expressions previously derived with the approximate ones discussed in [1]. Specifically, we compare the moment expressions in (17) and (18) with

$$
\begin{aligned}
& m_{1}=\frac{B(n+1, \nu-1)}{\gamma B(n, \nu)} \\
& m_{2}=\frac{B(n+2, \nu-2)}{\gamma^{2} B(n, \nu)}
\end{aligned}
$$

where $\gamma=2 n /(\mu(1+\rho))$ and $B(a, b)=\Gamma(a) \Gamma(b) / \Gamma(a+b)$, which are the corresponding approximate expressions given in [1]. The numerical comparisons of (17) versus (19) and of 
(18) versus (20) are shown in Figs. 1 and 2, respectively. It is clear that there is a substantial difference between the exact and approximate expressions.

\section{CONCLUSION}

We have derived explicit expressions for the pdf, cdf, and the moments of multilook magnitude as well as an elementary expression for the marginal pdf of interferogram's phase. We have also provided a numerical comparison of these expressions with the approximate ones suggested by Gierull [1]. We expect that these new results will be of use with respect to modeling SAR interferograms.

\section{REFERENCES}

[1] C. H. Gierull, "Statistical analysis of multilook SAR interferograms for CFAR detection of ground moving targets," IEEE Trans. Geosci. Remote Sens., vol. 42, no. 4, pp. 691-701, Apr. 2004.

[2] A. P. Prudnikov, Y. A. Brychkov, and O. I. Marichev, Integrals and Series, vol. 2. Amsterdam, The Netherlands: Gordon and Breach, 1986.

[3] _ Integrals and Series, vol. 3. Amsterdam, The Netherlands: Gordon and Breach, 1986.

[4] N. L. Johnson, S. Kotz, and N. Balakrishnan, Continuous Univariate Distributions, 2nd ed.,vol. 1 New York: Wiley, 1994. 